\title{
Comentario de Santo Tomás de Aquino \\ al tratado De divinis nominibus de Dionisio Areopagita
}

\section{Capítulo I \\ Sobre la intención del texto y la transmisión de los nombres divinos \\ Lección $1^{1}$}

Commentary by Santo Tomás de Aquino

to the treatise De divinis nominibus by Dionisio Areopagita

\section{Chapter I}

On the intention of the text and the transmission of divine names

Lesson 1

\author{
Abel Miró i Comas² \\ Universitat de Barcelona
}

Recibido: 14.12 .2020

Aceptado: 28.12.2020

En este libro, que se titula «Sobre los nombres divinos [De divinis nominibus]», siguiendo la costumbre de aquellos que han transmitido la ciencia magistralmente [artificiose], Dionisio empieza, en primer lugar, presentando algunas consideraciones necesarias para todo el estudio

\footnotetext{
${ }^{1}$ Referencia del texto original: SANTO TOMÁs, In librum beati Dionysii De divinis nominibus expositio (Ed. Ceslai Pera, O. P.), Marietti, Turín-Roma, 1950, pp. 4-13.

2 abel.miro@ub.edu https://orcid.org/0000-0003-2239-308X

Volumen 1. Número 2. Julio - Diciembre 2021 
sucesivo, y, en segundo lugar, prosigue exponiendo el objeto principal [de su tratado] en el capítulo tercero.

En relación a lo primero, realiza dos consideraciones: en la primera, muestra la razón de los nombres divinos [rationem divinorum nominum]; y en la segunda, manifiesta que los nombres que trata en este libro son comunes a toda la Trinidad. Esto último lo expone en el capítulo dos.

En relación a la primera de estas consideraciones, introduce dos observaciones: en la primera hace notar que continúa el libro precedente, en el cual, dirigiéndose al beato Timoteo, dice que: «después de las imágenes teológicas [post theologicas Hypotyposes]», esto es, después del estudio de las distinciones divinas en virtud de las cuales las personas, en la Trinidad, se distinguen entre sí, pasará «a la revelación [ad reserationem]», esto es, a la manifestación «de los nombres divinos», según sus posibilidades. Porque exponerlos perfectamente parece estar por encima de las posibilidades del hombre.

En la segunda observación, empieza a presentar algunas cosas necesarias para la obra presente. Introduce aquí dos consideraciones: en una, indica el modo de proceder [modum procedendi] en esta obra [el método]; esto es lo primero que hay que conocer en cualquier doctrina. Y en la otra [consideración], muestra cómo debe entenderse la razón [el concepto, rationem] de los nombres divinos que utiliza en este libro [lección II].

Y estas dos consideraciones están suficientemente expresadas en el título de este capítulo, que es: «Ésta es la intención del tratado», en referencia a la primera; y «ésta es la transmisión [traditio] de los Nombres divinos», en referencia a la segunda.

En la primera de estas consideraciones, hace dos cosas: en primer lugar, muestra los elementos a partir de los cuales se procede en esta obra; y en segundo lugar, aquellas cosas que deben transmitirse.

[El método utilizado: una ley revelada]

En relación al primer punto, realiza dos cosas: en primer lugar, muestra cuáles son aquellas cosas a partir de las cuales se debe proceder en esta obra, que no debe respaldarse en la razón humana, sino en la Revelación, recogiendo lo que dice el Apóstol: «No en las palabras 
persuasivas de la sabiduría humana [non in persuasibilibus humanae sapientiae verbis] [I Co 2, 4] [...], sino en la doctrina del Espíritu, expresando las cosas espirituales con términos espirituales [sed in doctrina spiritus, spiritualibus spiritualia comparantes] [13]». Por eso dice Dionisio: «Ahora, la ley de las palabras [lex eloquiorum]», es decir, la ley que es transmitida en las Sagradas Escrituras, «será predefinida», es decir, predeterminada «por nosotros», como lo fue en otro tiempo por el Apóstol; esta ley es: «nosotros mostramos [asseverare]», o sea, afirmamos o proponemos, «la verdad de las cosas dichas sobre Dios no con palabras persuasivas de la humana sabiduría», es decir, [nosotros no proponemos la verdad de las Sagradas Escrituras con argumentos] que no se respaldan para probar su objeto, como de sus principales medios, sobre los principios de la sabiduría humana, que procede según la razón natural, «sino en la demostración derivada de la virtud [la fuerza, el poder, la capacidad] de los Teólogos», es decir, de aquellos que han transmitido la Escritura canónica, a saber, los Apóstoles y los Profetas; «la virtud» la entiendo como «movida por el Espíritu Santo [motae a Spiritu Sancto]». En efecto, en su doctrina, Dionisio se basa en la autoridad de las Sagradas Escrituras, cuyo vigor [robur] y poder [virtutem] se deriva de que los Apóstoles y los Profetas han sido movidos a hablar [moti sunt ad loquendum] por el Espíritu Santo, que se revelaba a ellos y hablaba en ellos [eis revelante et in eis loquente].

\section{[Justificación del método]}

En segundo lugar, Dionisio ofrece la razón de la ley expuesta. La fuerza [virtus] de su argumento es la siguiente: en estas doctrinas [las que corresponden a un saber accesible para nuestra razón], podemos basarnos en los principios de la sabiduría humana, gracias a los cuales pueden enseñarse o transmitirse [traduntur] aquellas cosas que son cognoscibles y decibles [cognoscibilia et dicibilia] por los hombres, cosas que, a su vez, pueden ser conocidas y dichas [cognosci et dici] por aquellos que poseen ya la doctrina [o ciencia] acerca de ellas.

Pero en el ámbito de la doctrina de la fe [doctrina fidei], en cambio, se proponen a los hombres algunas cosas ignoradas e indecibles [ignota et indicibilia] a las cuales se adhieren los que tienen fe, sin estar en condiciones de conocerlas o explicarlas perfectamente; sin 
embargo, se adhieren a estas cosas con más certeza y con una adhesión más fuerte que a cualquier conocimiento natural.

En la doctrina de la fe, por lo tanto, no podemos basarnos en los principios de la sabiduría humana.

Esto es lo que dice Dionisio: «por la cual», es decir, gracias a la fuerza [virtutem] de la Revelación que procede del Espíritu Santo en los Apóstoles y en los Profetas, nosotros, por la fe, «nos unimos con las cosas inefables e ignoradas [coniungimur innefabilibus et ignotis]», o sea, a la verdad divina que excede toda palabra y todo pensamiento del hombre. La fe no se une con estas cosas de modo que haga al hombre creyente conocer y hablar de ellas como son, porque esto sería propio de la visión abierta [apertae visionis], sino que se une a ellas «inefablemente e ignotamente [ineffabiliter et ignote]»: «Porque ahora vemos como a través de un espejo [videmus enim nunc per speculum]», como se dice en Corinth. 13 [12].

Y porque nadie menospreciara esta unión [la de la fe con las verdades divinas] a causa de su imperfección, añade [que se produce] «según una unión superior a nuestra virtud y operación racional e intelectual [secundum meliorem unionem nostrae rationalis et intellectualis virtutis et operationis]», esto es, superior a la virtud [virtutem] y a la operación de nuestra razón y entendimiento; aquí Dionisio usa el genitivo en lugar del ablativo, como hacen los griegos.

Nosotros, mediante la fe, nos unimos [coniungimur] a verdades más altas que aquellas que nuestra razón natural puede alcanzar, y nos adherimos a ellas con mayor certeza que a las otras, pues la Revelación divina posee una certeza mayor que cualquier conocimiento humano. Dionisio distingue entre «racional e intelectual»; veamos el porqué: algunas cosas las conocemos naturalmente, es decir, las contemplamos inmediatamente [per se] sin necesidad de investigación alguna, y eso es propio del entendimiento [intellectus]; otras cosas, en cambio, son conocidas por medio de una investigación [per inquisitionem], y son propias de la razón [ratio]. Distingue, además, entre «nuestra operación y virtud [operationis et virtutis]», porque muchas cosas, sobre las cuales no pensamos [speculemur] actualmente, las conocemos de manera virtual [virtute] [esto es, en tanto que poseemos la capacidad activa para llegar a conocerlas].

Volumen 1. Número 2. Julio - Diciembre 2021 
Después, muestra qué cosas deben enseñarse en esta doctrina; en primer lugar, expone la afirmación o tesis [propositum] y, a continuación, la demuestra.

[Lo que debe enseñarse: los nombres divinos presentes en las Sagradas Escrituras]

Los principios a partir de los cuales procede esta doctrina son recibidos por Revelación del Espíritu Santo, y están contenidos en las Sagradas Escrituras; por este motivo — concluye Dionisio - de ningún modo debemos osar «decir con la boca o pensar algo acerca de la Divinidad supersubstancial [Deitate supersubstantiali]». La Divinidad está por encima de toda substancia, y, por esto, permanece oculta para nosotros —únicamente las substancias creadas están proporcionadas a ser conocidas [proportionatae ad cognoscendum] y a ser expresadas [ad loquendum] por nosotros-, «exceptuando lo que se nos ha revelado divinamente [sobre Ella] por medio de las palabras sagradas [ex sanctis eloquiis]», esto es, lo que ha sido expresado a través de las palabras sagradas [per sancta eloquia]. Expresamente no dice: «en $[i n]$ las palabras sagradas», sino «por medio [ex] de las palabras sagradas», porque todas aquellas cosas que pueden extraerse a partir de aquellas otras que están contenidas en las Sagradas Escrituras no son extrañas a esta doctrina, aunque no estén contenidas [explícitamente] en las Escrituras.

Después demuestra la tesis antes afirmada: en primer lugar, expone el argumento y, en segundo lugar, prueba aquello que está presupuesto en la demostración.

[El argumento]

La fuerza [virtus] de su demostración [rationis] es la siguiente: sobre aquello que únicamente sabe [scitur] un único individuo, nadie puede pensar ni decir algo, a menos que le haya sido manifestado por él. Pero solamente a Dios le conviene conocerse perfectamente a sí mismo según aquello que es [es decir, según su esencia] [perfecte cognoscere seipsum secundum id quod est]. En consecuencia, nadie puede decir o pensar algo con verdad [vere],

Volumen 1. Número 2. Julio - Diciembre 2021 
a menos que le sea revelado por el mismo Dios. Y la divina Revelación, sin duda, está contenida en las Sagradas Escrituras.

Por este motivo, dice Dionisio que «conviene atribuir a Él», a saber, únicamente a Dios, «la ciencia supersubstancial de la ignorancia de la supersubstancialidad [supersubstantialem scientiam ignorantiae supersubstantialitatis]», es decir, de la supersubstancialidad divina ignorada; esta supersubstancialidad no es ignorada en virtud de algún defecto suyo, sino en virtud de su exceso y sobreabundancia [excessum], porque está «por encima de la razón y del entendimiento» creados y por encima de «la misma substancia» creada, que es el objeto conmensurado al entendimiento creado, como la esencia increada está proporcionada a la ciencia increada. Y, por esta razón, como la esencia divina es supersubstancial, [Dionisio] llama «supersubstancial», también, a su esencia. En efecto, siempre es necesario que el objeto de la facultad cognoscitiva [obiectum cognitivae virtutis] esté proporcionado a la facultad del cognoscente [virtuti cognoscenti].

Sin embargo, para que no permanezcamos completamente en la ignorancia de Dios [in Dei ignorantia], añade: es oportuno que nosotros, «dirigiendo la mirada [respicientes]», a través de la contemplación espiritual, «hacia lo alto [ad superius]», esto es, hacia lo que está por encima de nosotros — hacia Dios—, tendamos «hacia el rayo de las palabras teárquicas que Él mismo envía [radius thearchicorum eloquiorum seipsum immittit]»; es decir, [hacia el rayo] que se extiende, que se propaga, «hacia los esplendores superiores [ad superiores splendores]», que son las verdades inteligibles sobre las cosas divinas [ad veritates intelligibiles divinorum].

La verdad de las Sagradas Escrituras es como una cierta luz a manera de rayo derivado de la Verdad Primera [quoddam lumen per modum radii derivatum a prima veritate], una luz, en efecto, que no se extiende como si, por ella, pudiéramos ver la esencia de Dios o conocer todas las cosas que Dios en sí mismo conoce o [que conocen] los ángeles y los bienaventurados, que ven su esencia, sino que, mediante la luz de las Sagradas Escrituras, se manifiestan las cosas inteligibles de Dios [intelligibilia divinorum] hasta un cierto límite o medida [terminum vel mensuram].

Y así, mientras nuestro conocimiento de Dios no se extienda más allá de lo que se extiende la luz de las Sagradas Escrituras [lumen Sacrae Scripturae], «estamos contraídos» y coartados 
por ciertos límites, envueltos por «la divina templanza y santidad»: «santidad», porque preservamos la verdad de las Sagradas Escrituras limpia [mundam] de cualquier error; «templanza», porque no ingerimos de las Sagradas Escrituras más de lo que estamos en condiciones de soportar [quam nobis est datum].

[Los presupuestos del argumento]

Más adelante, manifiesta los presupuestos de esta demostración: primero, que Dios solamente es conocido por sí mismo [Deus soli sibi sit notus], pero oculto para nosotros; y después, manifiesta el modo a través del cual el conocimiento divino nos es comunicado.

El primer punto lo aclara de dos modos: primeramente, con argumentos; en segundo lugar, con autoridades.

Propone dos argumentos; el primero es el siguiente: las cosas divinas son reveladas por Dios de acuerdo con la capacidad [secundum proportionem] de aquellos a quienes se revelan; pero conocer el infinito está por encima de la capacidad [supra proportionem] del entendimiento finito; en consecuencia, lo que Dios es [quod Deus est] no es conocido a partir de la Revelación divina por ningún entendimiento creado.

En relación a esto dice: «algunas cosas son reveladas» por Dios «y son vistas» por nosotros «según la capacidad de cada mente [secundum proportionem mentium uniuscuiusque]». Y esto lo afirma «si es que conviene creer en algo a la Teología», esto es, a las Sagradas Escrituras «sapientísimas y verísimas», porque se dice en Mateo 25, 15: «Dad ... a cada uno según su propia capacidad [dedit... unicuique secundum propriam virtutem]».

Propone dos motivos de los cuales se sigue que las Sagradas Escrituras son lo máximamente creíble [maxime credendum]. Es por dos razones, ciertamente, que no se cree a alguna persona: o bien porque es considerada ignorante [vel reputatur ignorans] o bien porque se la considera mendaz [mendax]. Ahora bien, como las Sagradas Escrituras son sapientísimas [omni-sapiens] y verísimas, en tanto que son reveladas y comunicadas por Dios, que es la Verdad y conoce a todas las cosas [omnia sciens], debe creerse a las Sagradas Escrituras en grado máximo.

Volumen 1. Número 2. Julio - Diciembre 2021 
Y habla de «teárquica [thearchica]» — esto es, divina— «bondad», separada de todo aquello que es «mensurado [mensuratis]» —o sea, finito- «por la inmensurabilidad [immensuratione]» - es decir, por la infinidad de la divina esencia [infinitate divinae essentiae]-, pero no porque no sea conocida en absoluto, sino porque no es comprehendida [non quidem ut nullo modo cognoscatur, sed ut non comprehendatur]. Y, en virtud de esto, añade: «como incomprehensible». En efecto, la esencia divina es alcanzada [attingitur] por la mente de los bienaventurados, pero no es comprehendida [comprehenditur]. Y esto lo hace Dios «con la justicia que salva [in iustitia salutari]». La esencia de la justicia distributiva consiste en dar a cada uno de acuerdo con su condición. Y así como, por medio del orden de la justicia distributiva establecida por el príncipe de la ciudad, se preserva [salvatur] todo el orden político, asimismo, gracias a este orden de la justicia, se preserva todo el universo, sin el cual todas las cosas se hundirían en la confusión [remaneret confusa]. Y esto [la teárquica bondad] lo hace «según conviene a Dios», porque conviene a Dios, por su bondad, salvar a aquellos que ha creado.

Presenta, a continuación, el segundo argumento [para probar que Dios solamente es conocido por sí mismo]: un grado de los entes no puede ser comprehendido por el inferior [superior gradus entium comprehendi non potest per inferiorem], como las cosas inteligibles no pueden ser comprehendidas perfectamente por las sensibles, ni las simples por las compuestas, ni las incorpóreas por las corporales; pero Dios está por encima de todo el orden de los existentes [super omnem ordinem existentium]; en consecuencia, no puede ser comprehendido por ninguno de los existentes.

Y por esto dice: «así como las cosas inteligibles son incomprehensibles e incontemplables [incomprehensibilia et incontemplabilia] por las que captan los sentidos», esto es, por las sensibles; «y las simples y carentes de figura [infigurabilia]» por aquellas que «tienen composición y figura», o sea, por aquellas que son compuestas y figuradas (la figura, ciertamente, solamente es propia de las cosas compuestas); y así como la «carencia»o privación de las formas corpóreas, propia de las realidades incorpóreas, que es, en efecto, una carencia o privación «intangible»e «infigurable», hace que las mismas realidades incorpóreas [ipsa incorporia], que carecen de forma y son intangibles e infigurables, «sean incomprehensibles e incontemplables por las cosas formadas según las figuras de los 
cuerpos», o sea, por los mismos cuerpos; asimismo, «según el mismo argumento de la verdad [rationem veritatis], se pone por encima la Unidad [superponitur Unitas], o sea, Dios», que es la misma Unidad, existente como uno por su esencia [quasi existens unus per suam essentiam], «la cual es supersubstancial y está por encima de las substancias y por encima de la mente [super mentem]», sobrepasando las mentes [mentibus], o sea, los espíritus intelectuales; «y [esta Unidad] es el mismo Bien», o sea, Dios, «que está por encima de la deliberación [super deliberationem]», por encima de la razón, «y es indeliberable respecto todas las deliberaciones [indeliberabile omnibus deliberationibus]», esto es, no es investigable por ninguna razón creada y «está por encima de la palabra [super verbum]», es decir, por encima de toda locución de la criatura [super omnem locutionem creaturae]: «es inefable», a saber, indecible por «cualquier palabra» creada.

En este pasaje toca cuatro cosas: las «sustancias», que son los objetos del conocimiento [obiecta cognitionis]; la «mente», a saber, el entendimiento simple [intellectum simplicem]; la deliberación, es decir, la razón investigadora [rationem inquirentem], que pertenece a la facultad cognoscitiva; y la «palabra [verbum]», que pertenece a la manifestación del conocimiento [ad manifestationem cognitionis]. Menciona estas cuatro cosas no solamente porque quiere mostrar que Dios no puede ser comprehendido por ninguna facultad cognoscitiva ni perfectamente manifestado por una locución, sino porque, además, quiere mostrar que no puede serlo por ningún objeto creado ni por ninguna semejanza creada. Por esto en los ejemplos que pone, no dice solamente que los inteligibles sean incomprehensibles por los sentidos, sino por las mismas cosas sensibles, porque a través de las cosas sensibles no es posible comprehender a las inteligibles. Y la misma razón puede aplicarse a los otros ejemplos.

Debe remarcarse que, según Dionisio, no solamente las cosas inteligibles son «incomprehensibles» por las sensibles, sino también «incontemplables», porque mediante aquello que es de orden inferior, no solamente no puede comprehenderse lo que es de orden superior, sino que tampoco puede contemplarse. En efecto, contemplamos [algo] a través de otro [per aliud] cuando, por medio de un objeto, podemos ver la esencia de otro y, así, saber qué es. Pero se comprehende su esencia cuando se conoce perfectamente cuanto en ella hay de cognoscible [perfecte cognoscitur, sicut cognoscibilis est]. Porque quien conoce una 
conclusión demostrable por medio de una argumentación probable, aunque en cierto sentido la contempla, sin embargo, no la comprehende, porque no alcanza el modo perfecto de su conocimiento.

En consecuencia, Dios es incomprehensible por todo entendimiento creado, porque está por encima de toda mente y razón [super omnem mentem et rationem], en tanto que hay más claritas de la verdad en su esencia - [ claritas $]$ que pertenece a su cognoscibilidad—, que en la virtud cognoscitiva de algo creado. Por esto, ninguna criatura puede alcanzar [pertingere] de modo perfecto el conocimiento de Él —conocimiento llamado «ciencia supersubstancial»-, pues alcanzarlo equivaldría a comprehender a Dios.

No obstante, el entendimiento creado puede contemplar la esencia de Dios, alcanzándola de algún modo [aliquo modo], pero no mediante algunos objetos [aliqua obiecta], especies [inteligibles] o cualesquiera semejanzas creadas, porque ninguna de estas cosas es capaz de conducirnos [manuducere] hasta la esencia divina - mucho menos de lo que un cuerpo lo es para conducirnos hasta la esencia incorpórea.

Así pues, según el argumento [rationem] de Dionisio, hay que decir que Dios es incomprehensible por todo entendimiento e incontemplable por nosotros en su esencia, mientras nuestro conocimiento permanezca atado a las cosas creadas, en tanto que son connaturales a nosotros [utpote nobis connaturalibus]; y esto es lo que ocurre en el estado de vía [in statu viae].

[El modo de significar de los nombres divinos]

Y como Dios es llamado unidad, para que nadie crea que es la unidad formalmente inherente en las cosas - como si fuera una unidad participada en las mismas cosas_-, Dionisio se refiere a Él como «la unidad» — subsistente por sí misma— «que unifica a toda unidad [unificans omnem unitatem]»-que difunde [diffundens] la unidad a todas las cosas que, de algún modo, participan de la unidad-. Como Dios es llamado unidad supersubstancial [unitatem supersubstantialem] y bien por encima de la mente [bonum super mentem], alguien podría creer que Dios de ningún modo puede llamarse substancia, mente o algo de este tipo y, 
por esto, para excluir esta opinión, afirma que Dios es «substancia», pero, al mismo tiempo, «supersubstancial».

Para poner eso de manifiesto, hay que considerar que los nombres, en tanto que son impuestos por nosotros, significan según el modo en que las cosas se presentan a nuestro conocimiento [significant secundum quod res in cognitionem nostram cadunt]. En consecuencia, como aquello que Dios es está por encima de nuestro conocimiento [sit supra cognitionem nostram], como ya ha sido mostrado, mientras que nuestro conocimiento está proporcionado [commensuretur] a las cosas creadas, los nombres impuestos por nosotros [a Dios] no significan según el modo que conviene [congruit] a la divina excelencia, sino según el que conviene a la existencia de las cosas creadas [secundum quod convenit existentiae rerum creatarum]. Pero el ser de las cosas creadas se deriva del ser divino según una semejanza imperfecta [esse autem rerum creatarum deductum est ab esse divino secundum quamdam deficientem assimilationem]. Por lo tanto, según la semejanza de Dios que, en cierto modo, se encuentra en las cosas creadas, los nombres impuestos por nosotros a Dios no pueden decirse de Él tal y como se aplican a las criaturas, sino por un cierto exceso [quemdam excessum]; a esto se refiere Dionisio al afirmar que Dios es una «substancia supersubstancial [supersubstantialis substantia]»; y semejantemente, cuando dice que es un «entendimiento no inteligible [intellectus non-intelligibilis» —es decir, no como los entendimientos que son entendidos por [intelliguntur] por nosotros-; «y que es una palabra no decible [verbum nondicibile]»—esto es, no como las palabras que son dichas [dicuntur] por nosotros.

Ahora bien, así como los nombres impuestos por nosotros pueden decirse de Dios gracias a aquella semejanza que las criaturas poseen respecto Dios, igualmente, en la medida que las criaturas son deficientes [deficiunt] en su representación de Dios, los nombres impuestos por nosotros pueden negarse [removeri] de Dios y predicarse sus opuestos. Por este motivo, Dionisio afirma que, así como Dios se llama Razón [Ratio], también puede llamarse «Irracionalidad [Irrationabilitas]»; y así como se llama Entendimiento [Intellectus], puede llamarse «No-inteligibilidad [Non-intelligibilitas]»; y así como se llama Palabra [Verbum], puede llamarse «Innominabilidad [Innominabilitas]»; y no porque Él carezca de estas perfecciones, sino porque «no es existente como los existentes [secundum nihil existentium est existens]», no existe del mismo modo que cualquier cosa existente; y, además, «Él es la 
causa del existir de todas las cosas [ipse quidem est causa existendi omnibus]», comunicando [transfundiens], de algún modo, su semejanza a cada realidad, para que así pueda ser dicho a partir de los nombres de las criaturas. Por eso, «Él es No-existente [Ipsum est non-existens]», no como carente de ser [deficiens ab essendo], sino como existente «por encima de toda substancia [supra omnem substantiam]»; y es Innominable en el sentido que «Él podría enunciarse a sí mismo propia y sabiamente [Ipsum de seipso proprie et scienter enuntiet]», esto es, según la propiedad de su ser [proprietatem sui esse] y según la perfecta ciencia de sí mismo [perfectam sui ipsius scientiam], pero que, de este modo, ninguna criatura está en condiciones de enunciarlo.

De todo lo que se ha dicho, Dionisio extrae la siguiente conclusión principal: «Por lo tanto, acerca de la Divinidad supersubstancial y oculta [supersubstantiali et occulta Deitate], no debe osarse decir ni pensar [dicere neque cogitare] nada que esté más allá de las cosas que, por inspiración divina, nos han sido manifestadas por medio de los libros sagrados»; esto es lo que ha expuesto.

[Los argumentos de autoridad]

Más adelante, aquello que antes se ha probado por medio de argumentos [filosóficos], se muestra por autoridad, cuando dice: «la Divinidad, en las Sagradas Escrituras, benévolamente se ha transmitido [tradidit] a Sí misma»—o sea, ha transmitido su bondad como verdad [bonitatem eius, ut veritatem] —; «ha transmitido que la ciencia y contemplación de Sí misma es inaccesible [invia] a todos los existentes», es decir, que ninguno puede acceder a Ella; no dice, ciertamente, que ningún existente puede acceder a cualquier género de ciencia o contemplación, sino a aquél por el cual [la Divinidad] se conoce o se contempla en su esencia [quod quid est], que es la ciencia comprehensiva de su substancia [scientia comprehensiva substantiae ipsius]. Y esta ciencia o contemplación es inaccesible, porque «está separada supersubstancialmente [est ab omnibus segregata supersubstantialiter]», esto es, según la eminencia [excessum] supersubstancial de la Divinidad. Efectivamente, sólo pertenece a la misma Divinidad el conocimiento de su esencia [quod quid est].

Volumen 1. Número 2. Julio - Diciembre 2021 
Y esto parece inferirse, principalmente, a partir de los textos siguientes: «Ningún hombre podrá verme y seguir con vida [non videbit me homo et vivet] [Ex 33, 20]»; «Él habita en la luz inaccesible, que ningún hombre ha visto ni puede ver [lucem inhabitat inaccessibilem, quem nullus hominum vidit sed nec videre potest] [I Tm 6, 16]». «Y verás que muchos autores sagrados [theologorum] han alabado a Dios no solamente como invisible e incomprehensible, sino también como inescrutable y no investigable: “¿Es que podrás comprehender los vestigios de Dios? [forsitan vestigia Dei comprehendes] [Jb 11, 7]?” ;“¡Qué incomprehensibles son sus juicios e inaccesibles sus caminos! [quam incomprehensibilia sunt iudicia eius et investigabiles viae eius]! [Rm 11, 33]"».

Y por qué razón se llama [a Dios] No-investigable, Dionisio lo explica de modo racional: «porque no existe ningún vestigio de aquellos que han llegado a su oculta infinidad [non existente ullo vestigio eorum qui transierunt ad occultam infinitatem ipsius]». En este texto —en el que hay una negación de más — se habla según la propiedad de la palabra: investigar [investigare], en efecto, significa ser conducido al término de la vía [ad viae terminum] a través de los vestigios [per vestigia] de alguno que va por la vía [alicuius euntis per viam]. Así pues, la Divinidad podría ser investigada, si alguno que hubiese llegado al conocimiento de Dios nos hubiera dejado algunos documentos — como ciertos vestigios o huellas-, para que nosotros pudiéramos llegar a ver a Dios. Pero esto no pasa: o bien porque nadie ha llegado hasta el final — si se habla de la visión comprehensiva-, o bien porque aquellos que han llegado a ver a Dios por esencia, como todos los bienaventurados, no han podido expresarnos la misma Esencia divina. Por esto Pablo, arrebatado [raptus] hasta el tercer cielo, dice que escuchó: «palabras misteriosas, que a ningún hombre le es permitido pronunciar [arcana verba, quae non licet homini loqui] ] [II Co 12, 4]».

De este modo, excluye tres modos de conocimiento: el primero es aquél por el cual una cosa es vista por sí misma [aliquid per seipsum videtur], y, en este sentido, se afirma que la Divinidad es «invisible [invisibilem]»; el segundo es aquél por el cual una cosa es conocida a través de una investigación de la razón [inquisitionem rationis], y, en este sentido, se afirma que la Divinidad es «inescrutable [inscrutabilem]», ya que escrutar implica una investigación [racional]; el tercero es aquél por el cual es conoce alguna cosa aprendiéndola de otra, y, por este motivo, se afirma que es «no-investigable [non-investigabilem $]$ » 
[La transmisión del conocimiento divino a los otros]

A continuación, muestra de qué modo el conocimiento de la Divinidad oculta se comunica a los otros. Sería contrario a la razón de Bondad divina, que [Ésta] retuviese su conocimiento para Sí misma sin comunicarlo a ningún otro, porque pertenece a la razón de bien el difundirse o comunicarse a los otros [de ratione boni sit quod se aliis communicet]. Y, por esto, aunque la ciencia supersubstancial de Dios sólo puede atribuirse a Dios, sin embargo, como Dios es el mismo Bien [ipsum Bonum], no puede ser que no se comunique a alguno de los existentes.

Pero el conocimiento [divino] no es comunicado a los otros del mismo modo que Dios se conoce a Sí mismo, sino «en tanto que Dios coloca [collocans]»-es decir, en tanto que conserva firmemente - «el rayo supersubstancial en Sí mismo de modo singular [singulariter in seipso supersubstantialem radium]» —esto es, en tanto que reserva para sí solo [sibi soli reservans] el conocimiento de su verdad supersubstancial [supersubstantialem veritatis suae cognitionem] — «se super-manifiesta [superapparet]», o, dicho de otro modo, se manifiesta desde lo alto [sursum apparet], «benignamente [benigne]»—o sea, no por necesidad sino por gracia [non ex necessitate sed ex gratia]—, «con iluminaciones adecuadas [proportionalibus illuminationibus]» — o proporcionadas— «a cada uno de los existentes [uniuscuiusque existentium]». Dicho más llanamente: pertenece a la razón de Bondad [divina] —reservándose para sí el modo de conocimiento que le es singular - comunicar a los inferiores por su gracia [ex sua gratia] algún tipo de conocimiento mediante sus iluminaciones, que se realizan de acuerdo con la proporción conveniente a cada uno [secundum proportionem uniuscuiusque].

Y no solamente super-manifiesta [superapparet] e ilumina, sino que también es gracias a Él que las inteligencias [mentes] inferiores, una vez iluminadas, hacen uso de la luz recibida [utentes dato lumine] para acercarse al conocimiento de Dios. Por este motivo, añade Dionisio: «[Dios] estimula a las inteligencias sagradas [sanctas mentes], en la medida les sea posible, a la contemplación divina [extendit sanctas mentes ad contemplationem ipsius possibilem eis]», porque, como ya se ha dicho, [Dios] es de algún modo contemplable para todos. Y como aquellos que contemplan a Dios, devienen, en cierto sentido [quodammodo], una sola cosa con Él (pues el entendimiento en acto [intellectus in actu] es, en cierto sentido, aquello que es entendido en acto [intellectum esse in actu]) y, por consiguiente, se asimilan [assimilantur] a 
Él en tanto que son informados por Él, sostiene Dionisio [que Dios también los estimula] «hacia la comunión y la asimilación».

[Las tres condiciones de las inteligencias santas]

Expone, finalmente, las condiciones de las inteligencias santas que llegan hasta Dios. La primera es que [esas inteligencias], en la medida que les es lícito por concesión divina y en la medida que les es conveniente según su propia condición, se lanzan a Él [ad Ipsum ingerunt]. Por este motivo dice: «aquellas inteligencias que tienden santamente según les es lícito y según les conviene a Él»—esto es, a Dios—, «no presumen» — a causa de la soberbia— de estar unidas con lo superior, es decir, con lo que está por encima de lo que les ha sido dado «convenientemente» según la manifestación o revelación de Dios; además, tampoco resbalan hacia abajo, hundiéndose por debajo de aquello que les ha sido concedido, «por la sujeción a las cosas peores [ex subiectione ad peius]», o sea, a causa de una cierta pusilanimidad, por la cual abandonadas las cosas mejores se adherirían a las peores.

La segunda condición de las santas inteligencias es que «firmemente e indeclinablemente se dirigen hacia el rayo super-resplandeciente [radium supersplendentem] de Dios», esto es, hacia la verdad que les viene manifestada desde lo alto [desuper]; así, la firmeza se refiere a la certeza y la indeclinabilidad, a la inmovilidad.

La tercera condición es que exhiban un afecto de amor [affectum amoris] por lo que Dios les manifiesta; así deben interpretarse esas palabras: «el amor proporcionado a las convenientes iluminaciones [commensurato amore convenientium illuminationum]», es decir, que su afecto persista alrededor de aquellas cosas que les han sido dadas según la medida conveniente, [aquellas cosas] gracias a las cuales «se elevan» a la realidad divina mediante alas espirituales, es decir, mediante contemplaciones intelectuales, «con una reverencia santa, casta y sagrada»: «con reverencia», en tanto que se abstienen de aquello que está por encima de ellas; «castamente», en tanto que no se detienen en las cosas inferiores; y «santamente», en tanto que se adhieren firmemente a aquellas cosas que les son dadas según la divina ordenación. 
REVISTA INTERNACIONAL DE FILOSOFÍA TEÓRICA Y PRÁCTICA

Volumen 1. Número 2. Julio - Diciembre 2021 ISSN: 2745-0333 (En línea) 